\title{
Caracterização de Polietilenos Lineares de Baixa Densidade II. Fracionamento por Cristalização Isotérmica a Partir do Estado Fundido
}

\author{
Antonio C. Quental, Lilian S. Hanamoto, Maria I. Felisberti \\ Instituto de Química, UNICAMP
}

\begin{abstract}
Resumo: Os polietilenos lineares de baixa densidade (PELBD) são uma classe de polietilenos com cadeias lineares contendo somente ramificações de cadeia curta devido à inserção de uma $\alpha$-olefina durante as reações de copolimerização com o eteno. As $\alpha$-olefinas comumente utilizadas são o 1-buteno, o 1-hexeno e o 1-octeno. Dependendo da $\alpha$-olefina e do catalisador utilizado na polimerização, os PELBD apresentam microestruturas que resultam em diferentes propriedades térmicas e mecânicas. Uma técnica simples e eficaz para a avaliação da distribuição de comonômeros nas cadeias poliméricas é o fracionamento por cristalização isotérmica a partir do estado fundido realizado via DSC. Neste método submete-se o polímero a diversas cristalizações isotérmicas durante o resfriamento, a partir do estado fundido. Este processo favorece a separação da fração cristalina em grupos contendo lamelas de diferentes espessuras, dependendo da distribuição da $\alpha$-olefina ao longo da cadeia do polímero e da massa molar. Durante o posterior aquecimento das amostras fracionadas observam-se picos endotérmicos em número igual ao de isotermas, que fornecem informações sobre a distribuição relativa dos comonômeros ao longo das cadeias dos PELBD. Neste trabalho, esta metodologia foi aplicada a diversos tipos de PELBD obtidos utilizando-se diferentes tipos e teores de $\alpha$-olefina, e diferentes sistemas catalíticos. A influência das condições experimentais sobre a eficiência do fracionamento também foi avaliada. A eficiência do fracionamento depende das temperaturas de cristalização e dos intervalos de temperaturas de cristalização utilizados, sendo que os tempos e as temperaturas e intervalos devem variar de acordo com a microestrutura do PELBD.
\end{abstract}

Palavras-chave: Polietileno linear de baixa densidade, microestrutura, DSC, fracionamento.

\section{Linear Low-Density Polyethylene Characterization II. Fractionation by Multiple-Step Isothermal Crystallization from the Melting State.}

Abstract: Linear low-density polyethylenes (LLDPE) are a class of polyethylenes with linear chains containing only short chain branches due to the insertion of $\alpha$-olefin units during the copolymerization with ethene. The $\alpha$-olefins commonly used are 1-butene, 1-hexene and 1-octene. Depending on the $\alpha$-olefin and the catalyst used for the polymerization, LLDPE presents different microstructures which determine the thermal and mechanical properties. One simple and efficient method to evaluate the microstructure of LLDPE is the fractionation by Multiple-Step Isothermal Crystallization from Melting State conducted by DSC. This method is based on several steps of isothermal crystallization of the polymer on decreasing the temperature from the melt. This process favors the separation of the crystalline material into groups having different lamellae thickness depending on the amount and distribution of the $\alpha$-olefin units in the macromolecular chains and on the molar mass. The melting endotherm of a fractionated sample is made up of the same number of peaks as the isothermal crystallization steps, which inform the relative comonomers distributions between different LLDPE chains. In this work, this methodology was applied to determine the relative comonomers distribution of different LLDPE. The isothermal temperature, temperature range and the time influence the efficiency of the fractionation and these parameters must be chosen according to the LLDPE microstructure.

Keywords: Linear low density polyethylene, characterization, DSC, fractionation.

\section{Introdução}

Os polietilenos lineares de baixa densidade (PELBD) apresentam propriedades que variam amplamente em função da concentração e tipo de comonômero e do catalisador utilizado na copolimerização. Uma vez que a distribuição do comonômero ao longo da cadeia dos PELBD é depen- dente do tipo de catalisador, o conhecimento desta distribuição leva a um entendimento mais detalhado dos mecanismos de polimerização, do grau de reatividade dos comonômeros, além do comportamento das cadeias com diferentes quantidades de comonômero frente à fusão e à cristalização, uma vez que estas refletem o comportamento global dos PELBD ${ }^{[1-3]}$.

A influência do tipo de catalisador na microestrutura

Autor para correspondência: Maria Isabel Felisberti, Grupo de Pesquisa em Polímeros (GPPol), Instituto de Química, UNICAMP, Caixa Postal 6154, CEP:13083-970, Campinas, SP. E-mail:misabel@iqm.unicamp.b 
dos PELBD tem sido investigada através do fracionamento destes polímeros, aliado a técnicas como: a cromatografia de permeação em gel (GPC), a ressonância magnética nuclear de carbono-13 $\left(\mathrm{RMN}^{13} \mathrm{C}\right)$, a espectroscopia infravermelho e a calorimetria diferencial de varredura (DSC). O GPC permite a determinação da massa molar média, o $\mathrm{RMN}^{13} \mathrm{C}$ e a espectroscopia infravermelho permitem a determinação da composição global do PELBD. Entretanto, estas técnicas não permitem uma avaliação mais refinada de como os comonômeros se distribuem ao longo das cadeias ${ }^{[1-7]}$.

Uma técnica muito utilizada para a determinação da distribuição dos comonômeros ao longo das cadeias é o fracionamento por eluição com gradiente de temperatura $(\mathrm{TREF})^{[6]}$. Esta técnica baseia-se no princípio de que cristais com lamelas de menor espessura fundem e dissolvem, em um dado solvente, a temperaturas menores. Assim, em um experimento de dissolução, cuja temperatura é progressivamente aumentada, é possível dissolver e, posteriormente, separar as frações, cujos cristais possuem espessuras de lamela diferenciadas. Estas frações podem ser analisadas por GPC, RMN e espectroscopia infravermelho. Assim, para o PELBD submetido à TREF as diferentes frações coletadas apresentarão diferenças na massa molar média e na distribuição de massa molar e no teor e distribuição de comonômeros ${ }^{[6]}$.

A espessura da lamela depende do comprimento da cadeia polimérica e do teor e da distribuição das ramificações ao longo da cadeia. Por isso, um polímero semicristalino que apresenta lamelas com uma ampla distribuição de espessura, pode ser separado em diferentes frações solúveis a diferentes temperaturas ${ }^{[2-7]}$.

No fracionamento de PELBD produzidos por catalisadores Ziegler-Natta, conforme aumenta-se a temperatura, as frações obtidas apresentam como característica a redução na concentração do comonômero e o aumento na massa molar. As frações obtidas a baixas temperaturas possuem uma concentração maior de comonômero e menor massa molar. A análise por calorimetria diferencial de varredura (DSC) destas frações, indica que quanto maior a concentração do comonômero e menor a massa molar, menor é a temperatura de fusão e de cristalização, e menor é o grau de cristalinidade. Este comportamento é atribuído a uma destruição na organização estrutural do polímero, causado pelo comonômero, com consequente diminuição na espessura das lamelas ${ }^{[2-7]}$.

Balbontin e colaboradores ${ }^{[6]}$ realizaram o fracionamento por eluição com gradiente de temperatura de PELBD obtidos por catalisadores do tipo Ziegler-Natta (multi-sítios) e metalocênico (único sítio). E verificaram que os PELBD obtidos por catalisadores multi-sítios, apresentam heterogeneidade na distribuição dos comonômeros tanto intermolecular, quanto intramolecular. Os autores notaram que os catalisadores metalocênicos resultam em polietilenos que podem apresentar uma distribuição heterogênea na massa molar, mas nunca nos comonômeros. Ou seja, independentemente da massa molar, a cadeia polimérica sempre apresenta uma distribuição homogênea do comonômero.

Wilfong ${ }^{[7]}$ através de medidas de espalhamento de raios-
$\mathrm{X}$ a baixo ângulo (SAXS), verificou que o período longo (definido como a distância entre os centros de duas lamelas adjacentes) apresenta uma queda acentuada quando a quantidade de ramificações nas frações aumenta, constatando que as cadeias com maior quantidade de comonômero formam lamelas menos espessas.

As condições de cristalização e os mecanismos envolvidos na cristalização dos PELBD a partir do estado fundido influenciam a formação das frações cristalinas, que apresentam diferentes temperaturas de fusão. Baseando-se nesse princípio, é possível fracionar um polímero semicristalino através da cristalização isotérmica a partir do estado fundido utilizando-se técnica de calorimetria diferencial de varredura (DSC). O método consiste em induzir a cristalização isotérmica a diferentes temperaturas a partir do estado fundido e, em seguida, verificar o comportamento da fusão. Este método tem a vantagem de ser mais rápido e com custo muito menor na caracterização da microestrutura dos PELBD quando comparado ao fracionamento por eluição com gradiente de temperatura ${ }^{[1,2,8,9]}$.

Patsch ${ }^{[10]}$, em seu artigo de revisão, faz uma comparação entre as técnicas de caracterização de poliolefinas, incluindo GPC, FTIR, RMN e DSC, destacando o fracionamento por cristalização isotérmica em DSC como uma técnica simples e poderosa na análise de ramificações em PELBD.

Zhang e colaboradores ${ }^{[11]}$ determinaram a polidispersidade de PELBD através do fracionamento por cristalização isotérmica em DSC, comparando a temperatura de fusão de cada pico observado na curva de DSC com poliolefinas e hidrocarbonetos com massa molar conhecida. A área dos pi$\cos$ foi usada no cálculo da fração de cadeias de massa molar definida. Em um trabalho posterior ${ }^{[12]}$, estes autores usaram a microscopia de força atômica e verificaram que a morfologia dos cristais muda gradualmente à medida que a massa molar diminui, passando de lamelar a uma morfologia granular.

Chen e colaboradores ${ }^{[13]}$ compararam os resultados de fracionamento por cristalização isotérmica e fracionamento por resfriamento a taxa contralada e baixa $\left(0,08{ }^{\circ} \mathrm{C} / \mathrm{min}\right)$, ambos em DSC, para PELBD e concluíram que o primeiro método fornece um histograma bem definido de espessuras de lamela, enquanto o segundo fornece uma curva de DSC cujo perfil representa uma distribuição contínua, que permite uma análise preliminar e qualitativa da distribuição. Também compararam os resultados de um fracionamento por TREF e por cristalização isotérmica, concluindo que estes são qualitativamente comparáveis.

Dentro deste contexto o objetivo deste trabalho é a avaliação da eficiência de diferentes condições de fracionamento por cristalização isotérmica a partir do estado fundido, assim como a aplicação da metodologia na determinação da microestrutura de diferentes tipos de PELBD.

\section{Experimental}

\section{Materiais Utilizados}

A Tabela 1 mostra a relação dos polietilenos utilizados, bem como as suas características químicas e físicas. Os polietilenos 
Tabela 1. Características físico-químicas dos PELBD utilizados.

\begin{tabular}{|c|c|c|c|c|}
\hline & PL 1880 & Dowlex 2045 & Elite 5400 & LFH 118 \\
\hline Catalisador & Metalocênico & Ziegler-Natta & $\begin{array}{l}\text { Ziegler-Natta / } \\
\text { Metalocênico }\end{array}$ & Ziegler-Natta \\
\hline Comonômero & 1-octeno & 1-octeno & 1-octeno & 1-hexeno \\
\hline Índice de Fluidez (g/10 min) ${ }^{a}$ & 1 & 1 & 1 & 1 \\
\hline $\bar{M}_{\mathrm{n}} \times 10^{-3}(\mathrm{~g} / \mathrm{mol})^{b}$ & 76 & 44 & 58 & 46 \\
\hline $\bar{M}_{w} \times 10^{-3}(\mathrm{~g} / \mathrm{mol})^{b}$ & 172 & 228 & 220 & 223 \\
\hline$\overline{\mathbf{M}}_{\mathrm{w}} / \overline{\mathbf{M}}_{\mathrm{n}} \mathrm{b}$ & 2,3 & 5,2 & 3,7 & 5 \\
\hline $\mathbf{T}_{\mathrm{c}}\left({ }^{\circ} \mathbf{C}\right)$ & 78 & 99 & 99 & 105 \\
\hline $\mathbf{T}_{\mathrm{f}}\left({ }^{\circ} \mathbf{C}\right)$ & 101 & 123 & 124 & 126 \\
\hline$\chi_{\mathrm{c}}(\%)$ & 31 & 46 & 41 & 47 \\
\hline d $\left(\mathrm{g} / \mathrm{cm}^{3}\right)^{a}$ & 0.902 & 0.920 & 0.916 & 0.920 \\
\hline Ramificação & hexila e longas & hexila & hexila & butila \\
\hline $\mathrm{CH}_{3} / 1000 \mathrm{C}^{\mathrm{c}}$ & 34 & 11 & 13 & 14 \\
\hline Nomenclatura $^{\mathrm{d}}$ & Oct-M & Oct-ZN & Oct-ZN/M & Hex-ZN \\
\hline
\end{tabular}

(a) dado fornecido pelo fabricante

(b) obtido por GPC em 1,2,4-triclorobenzeno a $140{ }^{\circ} \mathrm{C}^{[14]}$

(c) obtido por $\mathrm{RMN}^{13} \mathrm{C}^{[14]}$

(d) Nomenclatura utilizada ao longo deste trabalho

PL 1880, Dowlex 2045 e Elite 5400 foram produzidos pela Dow Chemical e o LFH 118 pela OPP. O PL 1880 pertence a família de PELBD conhecida como Afinnity, cuja característica principal é possuir uma mistura controlada de ramificações curtas e longas na cadeia principal. O Elite 5400 é produzido através da mistura dos catalisadores metalocênicos e Ziegler-Natta. Por último tem-se os PELBD Dowlex e LFH 118 que diferem somente no tipo de ramificação. A denominação adotada para os polietilenos neste trabalho indicam o tipo de monômero (Oct $=1$-octeno; Hex $=1$-hexeno $)$ e o catalisador utilizado na polimerização ( $\mathrm{M}=$ metalocênico; $\mathrm{ZN}=$ Ziegler-Natta). Assim os PELBD PL 1880, Dowlex 2045; Elite 5400 e LFH 118 são designados Oct-M, Oct-ZN, Oct-ZN/M e Hex-ZN, respectivamente, conforme mostrado na Tabela 1.

\section{Calorimetria Diferencial de Varredura (DSC)}

As medidas de DSC foram realizadas em um equipamento TA Instruments 2910. As amostras com massa na faixa de 5 a $10 \mathrm{mg}$ foram seladas em porta-amostras de alumínio, aquecidas a $10{ }^{\circ} \mathrm{C} / \mathrm{min}$ até $180^{\circ} \mathrm{C}$ e mantidas nesta temperatura por 5 minutos até a completa fusão de todos os cristalitos, apagando-se a história térmica do polímero. Em seguida, resfriou-se a uma taxa de $10{ }^{\circ} \mathrm{C} / \mathrm{min}$ até $0{ }^{\circ} \mathrm{C}$, mantendo-se nesta temperatura por 5 minutos, e então um novo aquecimento a $10^{\circ} \mathrm{C} / \mathrm{min}$ até $180^{\circ} \mathrm{C}$ foi realizado, obtendo-se assim as temperaturas de fusão $\left(\mathrm{T}_{\mathrm{f}}\right)$ e cristalização $\left(\mathrm{T}_{\mathrm{c}}\right)$, bem como as entalpias de fusão $\left(\Delta \mathrm{H}_{\mathrm{f}}\right)$ e de cristalização $\left(\Delta \mathrm{H}_{\mathrm{c}}\right)$. Os ensaios foram conduzidos em atmosfera de argônio, cujo fluxo foi mantido em $50 \mathrm{~mL} / \mathrm{min}$.

\section{Fracionamento por cristalização isotérmica a partir do estado fundido (FCIEF)}

O fracionamento por cristalização isotérmica a partir do estado fundido foi realizado no equipamento de DSC TA Instruments 2910. As amostras foram aquecidas a uma taxa de $10^{\circ} \mathrm{C} / \mathrm{min}$ até $160^{\circ} \mathrm{C}$ (primeira fusão) e deixada nesta temperatura por 5 minutos. Em seguida para o processo de cristalização, a amostra foi resfriada à mesma taxa até a primeira temperatura de cristalização isotérmica, e mantida nesta temperatura durante um tempo fixo (isoterma). Em seguida as amostras foram resfriadas rapidamente até a próxima temperatura de isoterma, com o mesmo tempo de espera. E assim, sucessivas cristalizações isotérmicas foram realizadas sempre com o mesmo intervalo de temperatura, até a temperatura final de isoterma. Em seguida a amostra foi resfriada até $0{ }^{\circ} \mathrm{C}$, e então aquecida a $10{ }^{\circ} \mathrm{C} / \mathrm{min}$ até $160^{\circ} \mathrm{C}$ (segunda fusão), obtendo-se assim a curva de DSC da amostra fracionada, com os vários picos de fusão correspondentes a cada fração de material cristalizado isotermicamente. Neste tipo de experimento, pode-se variar: (1) a temperatura da primeira isoterma e da última isoterma, ou seja, o intervalo de temperatura; (2) o tempo de cada isoterma; (3) o número de isotermas e (4) os saltos de temperatura entre uma isoterma e a subsequente. 


\section{Resultados e Discussão}

\section{Calorimetria diferencial de Varredura (DSC)}

Devido à distribuição heterogênea dos comonômeros ao longo da cadeia nos PELBD, ocorre um fenômeno comum que pode ser observado nas curvas de DSC: os PELBD apresentam pelo menos mais de um pico de fusão, atribuídos à presença de lamelas de espessuras diferentes.

$\mathrm{Na}$ Figura 1 encontram-se as curvas de DSC, normalizadas com relação a massa, para os PELBD, mostrando a etapa de resfriamento e o segundo aquecimento, todos a taxa constante de $10{ }^{\circ} \mathrm{C} / \mathrm{min}$. Nas curvas de DSC correspondentes ao resfriamento, Figura 1(a), observa-se para os quatro polietilenos um pico exotérmico relativo à cristalização, com uma cauda ampla a baixas temperaturas, que em alguns casos permite distinguir picos de baixa intensidade e ombros. As curvas correspondentes ao segundo aquecimento, Figura 1(b), apresentam picos endotérmicos relativos à fusão, os quais são largos sendo possível em alguns casos distinguir um segundo pico a menor temperatura. Por exemplo, um segundo pico pode ser observado nas curvas de DSC obtidas à taxa de $10{ }^{\circ} \mathrm{C} / \mathrm{min}$ para o Oct-ZN e Oct-ZN/M. Por outro lado, o Hex-ZN e Oct-M apresentam um pico relativamente largo. A entalpia de fusão, correspondente à área do pico endotérmico no segundo aquecimento, foi utilizada no cálculo do grau de cristalinidade $\left(\chi_{\mathrm{c}}\right)$, segundo a equação 1 .

$$
\% \chi_{c}=\frac{\Delta H_{f}}{\Delta H_{100 \%}} \times 100
$$

onde $\% \chi_{c}$ é o grau de cristalinidade em porcentagem, $\Delta \mathrm{H}_{\mathrm{f}}$ é a entalpia de fusão dos PELBD obtidas por DSC e $\Delta \mathrm{H}_{100 \%}$ é a entalpia de fusão para o PEAD $100 \%$ cristalino, cujo valor é igual a $293 \mathrm{~J} / \mathrm{g}^{[7]}$.

$\mathrm{Na}$ Tabela 1 encontram-se as temperaturas de cristalização $\left(T_{c}\right)$ e fusão $\left(T_{f}\right)$, tomados como as temperaturas correspondentes ao ponto de máximo e de mínimo do pico mais intenso relativo aos processos de cristalização e fusão, respectivamente, e o grau de cristalinidade $\left(\% \chi_{\mathrm{c}}\right)$. Observa-se que apesar da maior massa molar, a temperatura de fusão e de cristalização do PELBD Oct-M é menor que para as demais amostras, sendo este fato atribuído ao maior teor de comonômero. Os demais polietilenos diferem pouco em relação ao teor de comonômero e massa molar, mas as propriedades físicas diferem entre si. Por exemplo, o grau de cristalinidade é semelhante entre o PELBD Oct-ZN e PELBD Hex-ZN, porém o PELBD Hex-ZN apresenta uma $T_{f}$ maior. O PELBD Oct-ZN/M apresenta uma $T_{f}$ e $T_{c}$ e o perfil da curva de aquecimento semelhante ao PELBD Oct-ZN. Este conjunto de resultados refletem as diferenças microestruturais entre os quatro PELBD.

\section{Fracionamento por cristalização isotérmica a partir do estado fundido (FCIEF).}

$\mathrm{O}$ fracionamento por cristalização isotérmica a partir do estado fundido (FCIEF) foi conduzido com o objetivo de ca-
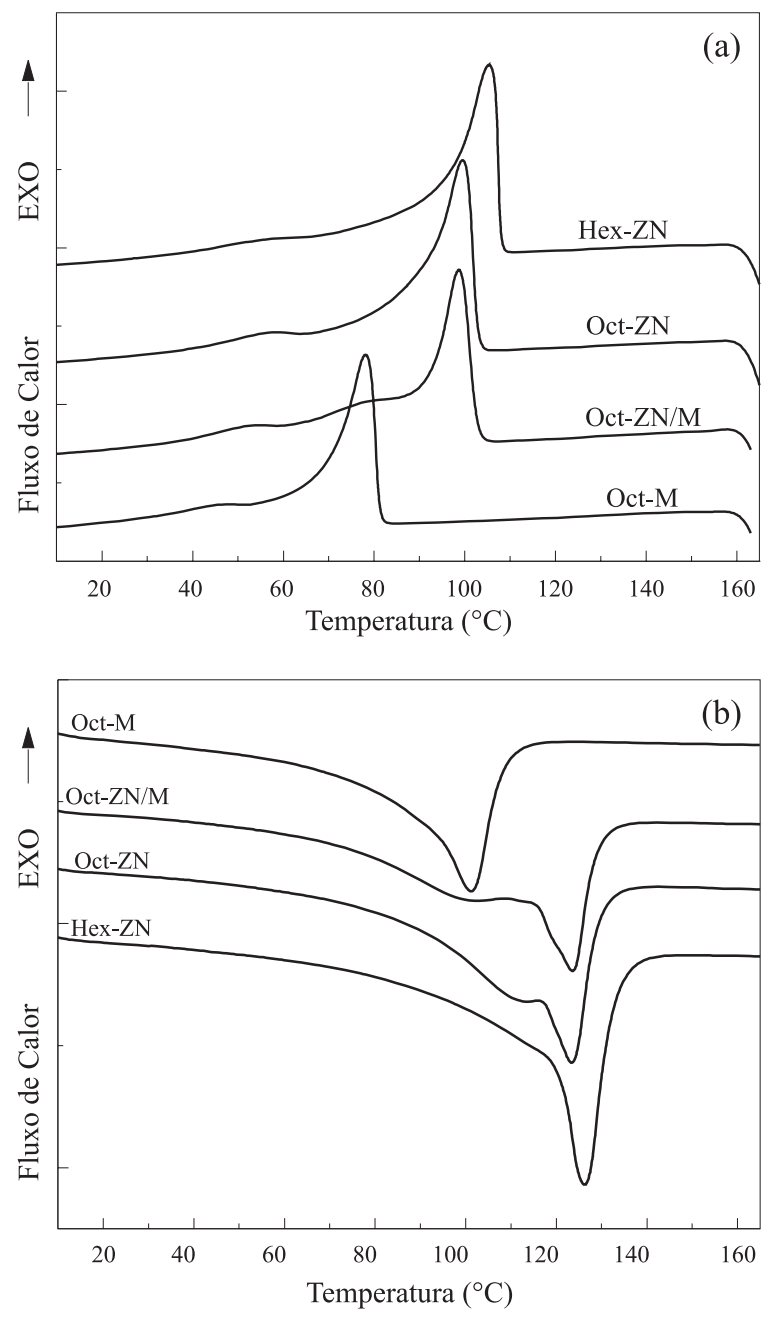

Figura 1. Curvas de DSC à taxa de $10{ }^{\circ} \mathrm{C} / \mathrm{min}$ obtidas sob atmosfera de argônio, normalizadas com relação a massa, para os diferentes LLDPE: a) resfriamento a partir do estado fundido; b) segundo aquecimento.

racterizar os PELBD quanto à microestrutura, ou seja, a distribuição dos comonômeros ao longo da cadeia. Este método permite que as cadeias do polímero contendo diferentes teores de comonômeros e massa molar, segreguem do polímero fundido, formando fases cristalinas com características específicas, tais como espessura de lamelas, e, portanto, temperaturas de fusão diferenciadas. O comonômero atua como um defeito na cadeia, ficando fora da estrutura cristalina. Assim, somente os segmentos de cadeia entre estes "defeitos" são passíveis de cristalização. Quanto menor o tamanho destes segmentos, menor será a espessura de lamela e, consequentemente, menor a temperatura de cristalização e de fusão. Por outro lado, as cadeias livres de comonômeros poderão cristalizar a maiores temperaturas formando cristais com lamelas mais espessas, que apresentarão maior temperatura de fusão ${ }^{[1-8]}$. O fracionamento por cristalização isotérmica a partir do estado fundido baseia-se em vários passos de cristalização isotérmica, através da diminuição da temperatura do polímero a partir do estado fundido. A subsequente curva de DSC no aquecimento mostra a fusão, que se manifesta em múltiplos picos, cujo número é o mes- 
mo dos passos da cristalização isotérmica.

Na Figura 2 encontram-se as isotermas de cristalização do PELBD Oct-ZN a diferentes temperaturas em duas condições experimentais distintas:

- Na condição I o número de isotermas é menor, porém o tempo é maior (120 minutos), sendo que a primeira isoterma é em $115^{\circ} \mathrm{C}$ e a última em $100^{\circ} \mathrm{C}$.

- Na condição II o número de isotermas é maior, porém o tempo é menor (20 minutos), sendo que a primeira isoterma inicia-se à temperatura de $130{ }^{\circ} \mathrm{C}$ e a última isoterma ocorre em $80^{\circ} \mathrm{C}$.

Na Figura 2a é mostrada a seqüência de isotermas a que o PELBD Oct-ZN foi submetido, condição I, e o fluxo de calor durante cada isoterma. Nota-se que o salto de uma isoterma a outra causa o surgimento de um pico na curva de fluxo de calor, que se deve apenas ao desequilíbrio térmico causado pela variação de temperatura. Na curva de fluxo de calor durante a isoterma a $115^{\circ} \mathrm{C}$ observa-se uma curva ascendente que na isoterma a $110^{\circ} \mathrm{C}$ tem um comportamento descendente, que está relacionada a exoterma de cristalização. Para as demais isotermas não se observa claramente picos exotérmicos, o que não significa a ausência de cristalização. Prova de que há cristalização fracionada é que a correspondente curva de fusão mostrada na Figura $2 \mathrm{c}$ apresenta múltiplos picos. Assim, provavelmente o calor envolvido nas demais isotermas é muito baixo relativamente à primeira cristalização. Esta hipótese encontra respaldo no fato de que a maior fração de PELBD que cristaliza a maior temperatura também funde a maior temperatura. (Figura 2c)

$\mathrm{Na}$ condição II de fracionamento, Figura $2 \mathrm{~b}$, a maior parte do polímero cristaliza a $115^{\circ} \mathrm{C}$, situação em que se observa o pico exotérmico de cristalização.

O pico mais intenso da fusão do Oct-ZN, submetido à cirstalização isotérmica na condição I (Figura 2c), ocorre a temperatura maior que o correspondente pico de fusão para a amostra cristalizada na condição II. Isto significa que o aumento do tempo de cristalização a $115^{\circ} \mathrm{C}$ de 20 minutos (condição II) para 120 minutos (condição I) resultou em cristais com lamelas mais espessas. O surgimento do pico acima de $117^{\circ} \mathrm{C}$, na curva de fusão da amostra submetida ao fracionamento pela condição I, destacado na figura $2 \mathrm{c}$, sugere que na isoterma a $110^{\circ} \mathrm{C}$ por 20 minutos tenha sido originado uma família de cristais, que sofreram reorganização durante a isoterma, resultando em cristais com lamelas mais espessas. Portanto, o tempo de isoterma é um fator importante no fracionamento. Tempos curtos podem não garantir que todas as cadeias ou segmentos de cadeias com potencial para cristalizar em uma dada temperatura participará da cristalização. Tempos demasiadamente longos podem induzir a outros processos, como a reorganização, podendo mascarar ou tornar mais complexa a análise.

$\mathrm{Na}$ condição I de cristalização não houve o fracionamento das cadeias menores e mais ricas no comonômero, pois se limitou à temperatura mínima de $100^{\circ} \mathrm{C}$. A curva de fusão da amostra submetida à condição II de cristalização mostrou
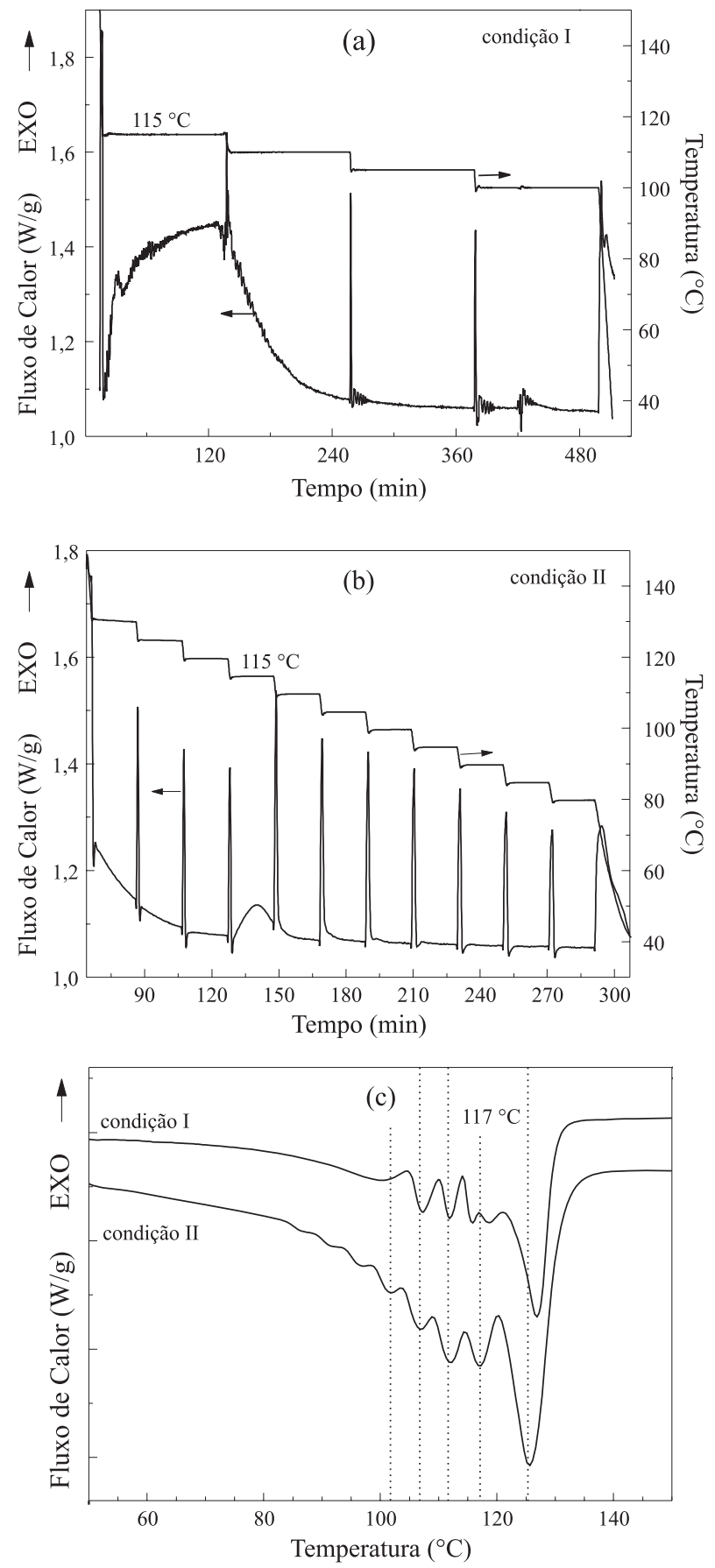

Figura 2. Fracionamento por cristalização isotérmica a partir do estado fundido aplicada ao Oct-ZN: (a) condição I; (b) condição II; (c) Fusão após o fracionamento. Curvas de DSC normalizadas com relação a massa.

picos pouco intensos a temperaturas menores que $100{ }^{\circ} \mathrm{C}$. Assim, a faixa de temperatura de cristalização também é um fator determinante da eficiência do fracionamento.

Na Figura 3 encontram-se as isotermas de cristalização e o comportamento de fusão do PELBD Oct-M, o polietileno de menor temperatura de fusão entre todos (Tabela 1), submetido a duas condições de cristalização (III e IV). A Figura 1(a) mostra que este polietileno cristaliza a partir de $80^{\circ} \mathrm{C}$, sob condições de resfriamento à taxa constante de $10^{\circ} \mathrm{C} / \mathrm{min}$.

As condições III e IV adotadas no seu fracionamento, apre- 
sentam o mesmo número de isotermas, porém as isotermas apresentam tempos e temperaturas de início diferentes:

- Na condição III cada isoterma tem duração de 120 minutos, sendo que a primeira isoterma ocorre a $110^{\circ} \mathrm{C}$, e a última a $95^{\circ} \mathrm{C}$ (quatro isotermas no total).

- Na condição IV cada isoterma tem duração de 90 minutos, sendo que a primeira isoterma ocorre a $100^{\circ} \mathrm{C}$, e a última a $85{ }^{\circ} \mathrm{C}$ (quatro isotermas no total).

Tanto na condição III (Figura 3a) como na IV (Figura 3b) observa-se que a curva de fluxo de calor durante a primeira isoterma é ascendente no início depois atinge um patamar que se mantem constante até a penúltima isoterma, quando apresenta um comportamento descendente. Isto sugere que ocorre cristalização durante quase todo o tempo do experimento e mesmo assim ocorre a separação de cadeias com massas molares e teores de comonômeros distintos.

A condição III de cristalização resultou em duas famílias principais de cristais que se fundem a $90^{\circ} \mathrm{C}$ e $105^{\circ} \mathrm{C}$ (Figura $3 b)$. É possível observar um ombro no pico com mínimo a $105^{\circ} \mathrm{C}$, que naturalmente de deve a uma família de cristais com espessura de lamela próxima à família que se funde a $105^{\circ} \mathrm{C}$. Já a condição IV de cristalização, resultou em quatro picos, porém o pico referente à fração de cadeias maiores e mais pobres em comonômero, ocorre à temperatura menor do que o seu correspondente pico na curva de fusão da amostra cristalizada na condição III. Assim, conclui-se que o fracionamento foi mais eficiente na condição IV em que as isotermas estão na faixa de temperatura em que a cristalização é cineticamente e termodinamicamente favorável. Ao contrário do observado na cristalização isotérmica do Oct$\mathrm{ZN}$, o tempo de cristalização para o Oct-M foi menos importante do que a faixa de temperatura, ou melhor, que a temperatura de início de cristalização.

Os PELBD Oct-ZN e Hex-ZN diferem por terem sido obtidos da copolimerização de eteno com os comonômeros 1-octeno e 1- hexeno, respectivamente, utilizando-se o mesmo tipo de catalisador (Ziegler-Natta). Porém, não diferem significativamente, no teor de ramificações, massa molar e sua distribuição e temperatura de fusão, Tabela 1 .

Na Figura 4 encontram-se os resultados obtidos através do fracionamento conduzido na condição II para os PELBD Oct-ZN e Hex-ZN. As condições de fracionamento e posterior aquecimento foram as mesmas para os dois polímeros.

As Figura $4 \mathrm{a}$ e $4 \mathrm{~b}$ apresentam as isotermas localizadas entre $130^{\circ} \mathrm{C}$ e $110^{\circ} \mathrm{C}$ (embora o fracionamento tenha sido conduzido entre $80^{\circ} \mathrm{C}$ e $130^{\circ} \mathrm{C}$ ). Para a cristalização do Oct$\mathrm{ZN}$, na isoterma a $115^{\circ} \mathrm{C}$ observa-se nitidamente um pico exotérmico largo (Figura 4b). Para as demais isotermas não se observa nenhum pico, não significando que não houve cristalização, como já discutido anteriormente. Isto mostra que provavelmente a maior parte deste polímero cristaliza durante o resfriamento em torno de $115^{\circ} \mathrm{C}$. Para o Hex-ZN, observa-se um pico exotérmico bem mais fino na isoterma a $115^{\circ} \mathrm{C}, \operatorname{logo}$ após o pico referente ao desequilíbrio térmico causado pelo salto entre as isotermas. Este fato já demonstra
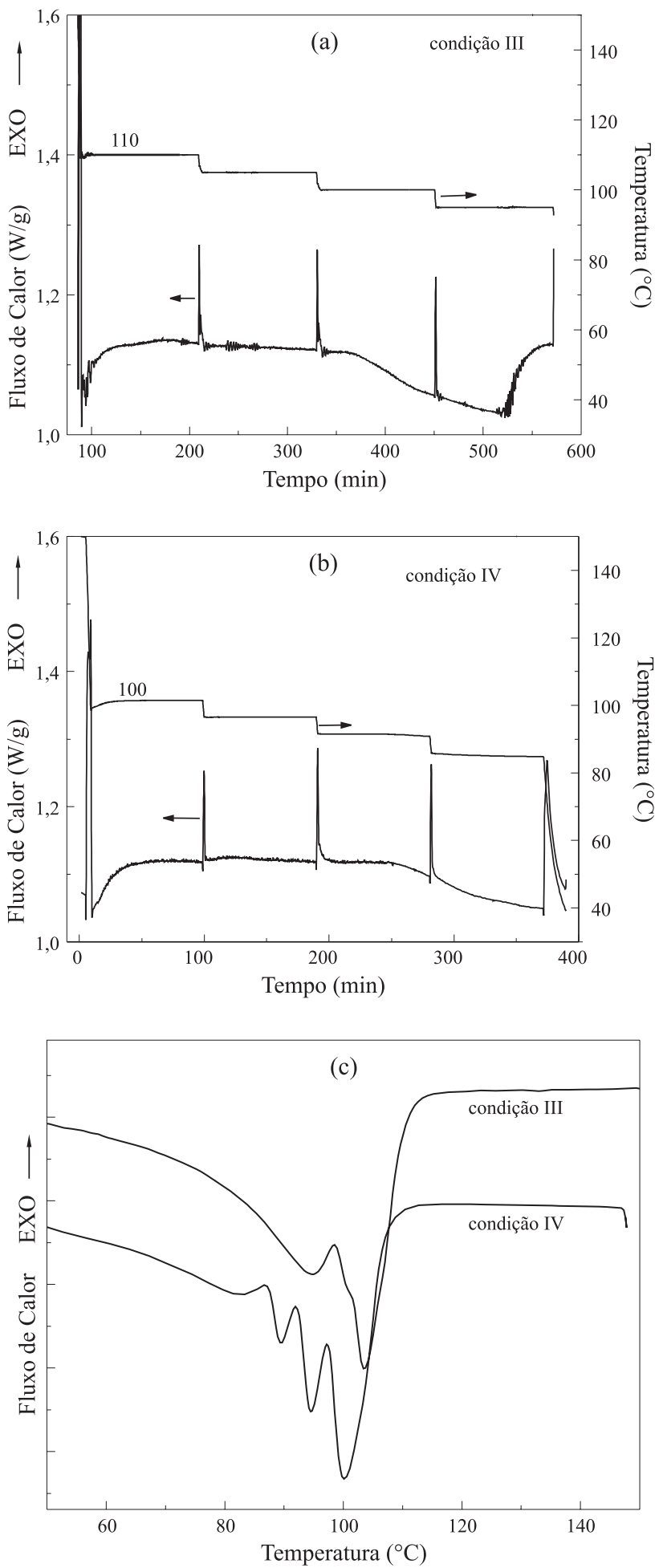

Figura 3. Fracionamento por cristalização isotérmica a partir do estado fundido aplicada ao Oct-M: (a) condição III; (b) condição IV; (c) Fusão após o fracionamento. Curvas de DSC normalizadas com relação a massa.

que a cinética de cristalização destes polímeros é diferente, apesar das massas molares e teores de comonômeros serem próximos.

A Figura $4 \mathrm{c}$ mostra os múltiplos picos de fusão resultantes do fracionamento. A fração de cristais de maior espessura do PELBD Hex-ZN formados por cadeias maiores e apresentan- 

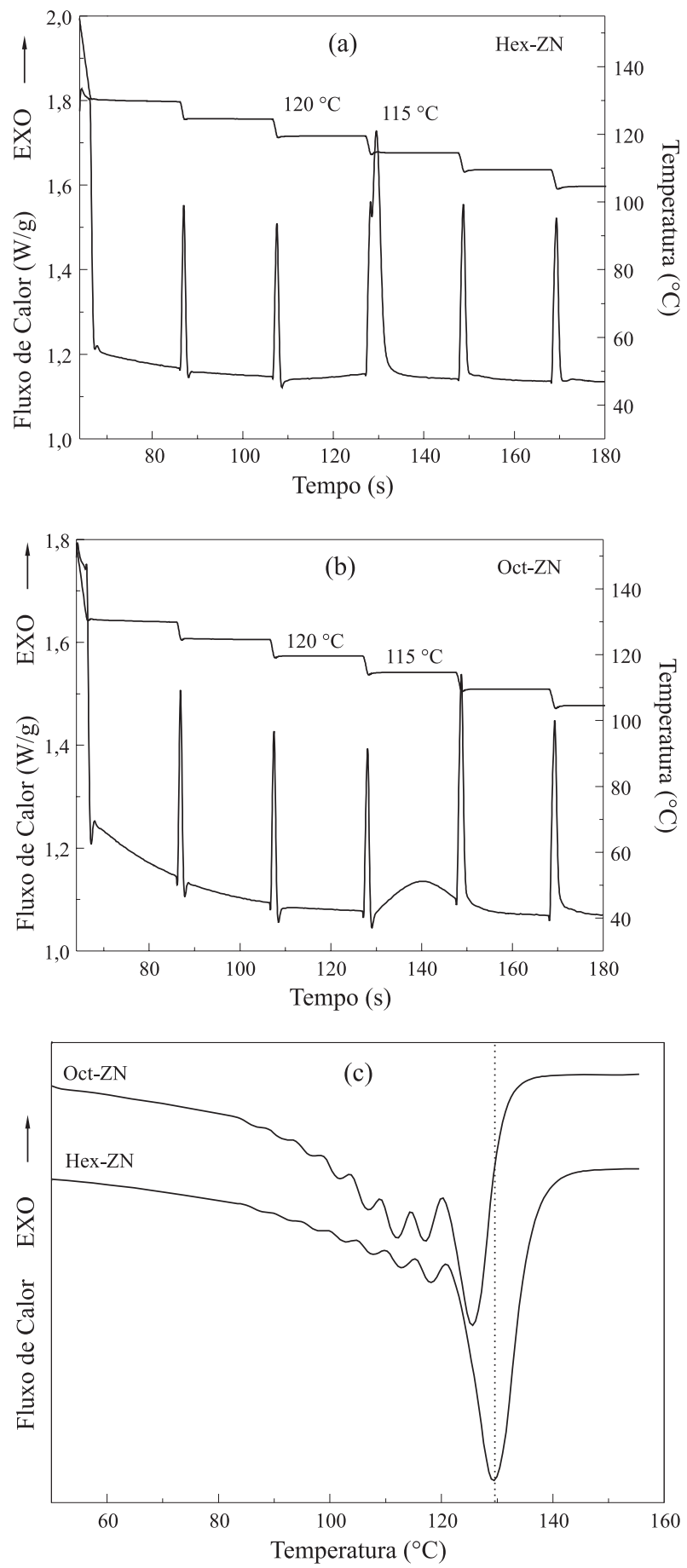

Figura 4. Fracionamento por cristalização isotérmica a partir do estado fundido. (a) aplicada ao Hex-ZN na condição II; (b) aplicada ao Oct-ZN na condição II; (c) Fusão após o fracionamento. Curvas de DSC normalizadas com relação a massa.

do menos ramificações, funde a temperaturas maiores, sendo o pico de fusão mais intenso comparativamente à fração correspondente do Oct-ZN. Uma vez que as condições de cristalização foram idênticas, os resultados levam à conclusão de que o Hex-ZN apresenta uma fração maior de cadeias maiores e mais pobres em ramificações que o Oct-ZN.

Na Figura 5 encontram-se as curvas de aquecimento após o fracionamento por cristalização isotérmica no estado fundido, para os PELBD contendo o 1-octeno como comonômero, cujas características encontram-se na Tabela 1 . Devido às diferenças microestruturais entre esses três polietilenos, as temperaturas de fusão e cristalização são diferentes e, portanto, não foi possível conduzir o fracionamento por cristalização isotérmica nas mesmas condições. Apesar disso, os resultados permitem uma comparação qualitativa entre as diferenças microestruturais dos polímeros, principalmente no caso do PELBD Oct-ZN e do PELBD Oct-ZN/M que foram fracionados na condição I. O PELBD Oct-ZN e o PELBD Oct-ZN/M apresentam perfis de fusão e de cristalização semelhantes (Figura 1). Entretanto, após o fracionamento há uma diferença significativa entre os dois polímeros (Figura 5). Por exemplo, o pico de fusão que ocorre a temperatura mais alta é mais intenso para o PELBD Oct-ZN. Isto significa que a fração de cadeias com o menor teor de comonômeros e maior massa molar é maior no caso do PELBD Oct-ZN. Também é possível inferir que a distribuição intermolecular de 1-octeno é mais homogênea para o PELBD Oct-ZN/M do que para o PELBD Oct-ZN.

O polietileno Oct-M foi submetido a condição IV de fracionamento, e apresenta um menor número de picos endotérmicos, apesar das condições I e IV apresentarem o mesmo número de isotermas de cristalização. Este resultado indica que a distribuição dos comonômeros ao longo das cadeias é mais homogênea para o PELBD Oct-M, o que é esperado para uma poliolefina sintetizada utilizando catalisador metalocênico. A menor temperatura de fusão apresentada por este PELBD deve-se à presença de ramificações longas, o que diminui a espessura das lamelas formadas.

O fracionamento por cristalização isotérmica utilizado no trabalho e o de fracionamento por solubilização a diferentes temperaturas (TREF) amplamente utilizado na caracterização de PELBD apresentam limitações similares. Não se pode garantir que somente cadeias com dimensões específicas cristalizem a uma dada temperatura, formando lamelas que se dissolverão (método TREF) ou fundirão (método FCIEF) a temperatura específica. Apesar disto, ambos os métodos ge-

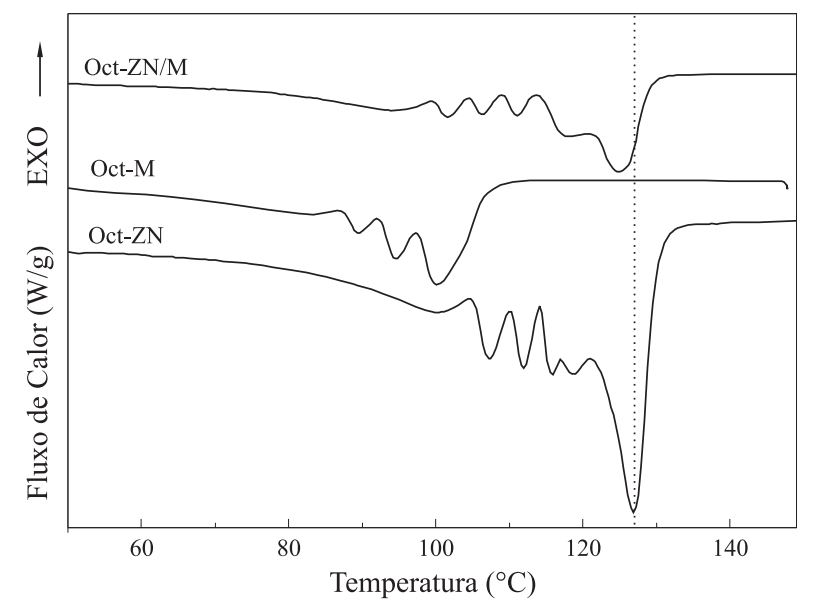

Figura 5. Curvas de Fusão obtidas após o fracionamento por cristalização isotérmica a partir do estado fundido aplicada aos PELBD contendo o 1octeno como comonômero. Curvas de DSC normalizadas com relação a massa. 
ram resultados que permitem diferenciar de forma qualitativa polietilenos lineares de baixa densidade com diferentes microestruturas Os resultados mostram isto claramente. Efeitos de recristalização e reorganização podem ocorrer durante o aquecimento, o que comprometeria a análise quantitativa, a qual não é objeto de estudo. No caso específico dos polietilenos estudados, exceção para o Oct-ZN, efeitos de reorganização e recristalização não foram significativos ou não comprometeram a análise relativa da microestrutura, pois foram observados picos de fusão em número igual ao de isotermas.

\section{Conclusões}

O fracionamento por cristalização isotérmica a partir do estado fundido é um método de análise simples e eficiente para caracterizar e comparar a microestrutura de PELBD onde o tipo, a distribuição e a concentração das ramificações influenciam a formação das lamelas cristalinas. Entretanto, a eficiência do fracionamento por cristalização isotérmica a partir do estado fundido depende das temperaturas de cristalização utilizadas e dos intervalos de temperaturas de cristalização. Os tempos e as temperaturas devem variar de acordo com a microestrutura do PELBD.

Através do fracionamento por cristalização isotérmica a partir do estado fundido, pode-se diferenciar os PELBD produzidos via catalisador Zieglar-Natta dos produzidos via catalisador metalocênico.

\section{Agradecimentos}

À FAPESP processos no 98/12239-6; 98/12238-0; 99/ 03642-4.

\section{Referências Bibliográficas}

1. Quental, A.C. - “ Blendas de Polietileno Linear de Baixa Densidade (PELBD) e Poli(propeno-co-eteno-co-1buteno)", Tese de Mestrado, Universidade Estadual de Campinas, UNICAMP, Brasil (2000).
2. Hanamoto, L. S. - "Estudo da Cinética de Cristalização de Polietileno Linear de Baixa Densidade: Influência da Microestrutura", Tese de Mestrado, Universidade Estadual de Campinas, UNICAMP, Brasil (2000).

3. Todo, A. \& Kashiwa, N. - Macromol. Symp. 101, p. 301 (1996).

4. Karbashewski, E.; Kale, L.; Rudin, A.; Tchir, W.J.; Cook, D.G. \& Pronovost, J.O. - J. Appl. Polym. Sci., 44, p. 425 (1992).

5. Neves, C.J.; Monteiro, E. \& Habert, A.C. - J. Appl. Polym. Sci., 50, p. 817 (1993).

6. Balbontin, G.; Camurati, I.; Dall'Occo, T. \& Zeigler, R. C. - J. Mol. Catalys. A : Chem., 98, p. 123 (1995).

7. Wilfong, D.L. \& Knight, G.W. - J. Polym. Sci. Part B: Polym. Phys., 28, p.861 (1990)

8. Fu, Q.; Chiu, F.C.; McReight, K.W.; Guo, M.; Tseng, W.W. \& Cheng, S.Z.D. - J. Macromol. Sci. Phys., B 36 (1), p.41 (1997).

9. Adisson, E.; Ribeiro, M.; Defieux, A. \& M. Fontanille Polymer; 33, p.4337 (1992).

10. Pasch, H. - Macromol. Symp., 165, p. 91 (2001)

11. Zhang, F.J.; Fu, Q.; Lu, T.J.; Huang H.Y.; He, T.B. Polymer, 43, p. 1031 (2002)

12. Zhang, F.J.; Fu, Q.; Lu, T.J.; Huang H.Y.; He, T.B. - J. Polym. Sci.: Polym. Phys., 40, p. 813 (2002).

13. Chen, F.; Shanks, R.A.; Amarasinghe, G. - Polym. Int., 53, P. 1975 (2004).

14. Quental, A.Q.; Hanamoto, L.S; Felisberti, M.I. Polímeros: Ciência e Tecnologia, 15 (4) p.274 (2005)

Enviado: 31/12/04

Reenviado: $16 / 06 / 05$

Aprovado: 28/06/04 